\title{
Controllable Coupling between Flux Qubits
}

\author{
Yu-xi Liu, ${ }^{1}$ L. F. Wei, ${ }^{1,2}$ J. S. Tsai, ${ }^{1,3}$ and Franco Nori ${ }^{1,4}$ \\ ${ }^{1}$ Frontier Research System, The Institute of Physical and Chemical Research (RIKEN), Wako-shi 351-0198, Japan \\ ${ }^{2}$ IQOQI, Department of Physics, Shanghai Jiaotong University, Shanghai 200030, People's Republic of China \\ ${ }^{3}$ NEC Fundamental Research Laboratories, Tsukuba Ibaraki 305-8501, Japan \\ ${ }^{4}$ MCTP, Physics Department, CSCS, The University of Michigan, Ann Arbor, Michigan 48109-1040, USA
}

(Received 27 June 2005; published 15 February 2006)

\begin{abstract}
We propose an experimentally realizable method to control the coupling between two flux qubits. In our proposal, the bias fluxes are always fixed for these two inductively coupled qubits. The detuning of these two qubits can be initially chosen to be sufficiently large, so that their initial interbit coupling is almost negligible. When a variable frequency or time-dependent magnetic flux (TDMF) is applied to one of the qubits, a well-chosen frequency of the TDMF can be used to compensate the initial detuning and to couple two qubits. This proposed method avoids fast changes of either qubit frequencies or the amplitudes of the bias magnetic fluxes through the qubit loops, and also offers a remarkable way to implement any logic gate, as well as tomographically measure flux qubit states.
\end{abstract}

PACS numbers: 85.25.Cp, 03.67.Lx, 74.50.+r

Introduction. - Superconducting Josephson junction circuits currently provide one of the best qubit candidates [1]. Quantum coherent oscillations and conditional gate operations have been demonstrated using two-coupled superconducting charge qubits [2]. Further, entangled macroscopic quantum states have been experimentally verified in systems of coupled flux [3], and phase [4,5] qubits.

Quantum computing requires that the interaction between different qubits can be selectively switched on and off. This is an extremely difficult and important issue. Several schemes have been proposed to realize controllable couplings and local qubit operations. One is a controllable coupling by dynamically tuning the qubit frequencies, e.g., in Refs. [5-9]. This tunable proposal requires that different qubits have the same frequencies (i.e., resonant interaction) when they are coupled. When they are decoupled, one of their frequencies should be suddenly changed by an external bias variable such that two-coupled subsystems have a larger detuning (i.e., nonresonant interaction). The second approach uses switchable couplings in charge qubit circuits by changing the bias magnetic flux, e.g., in Refs. $[10,11]$. In practice, the switching time of the magnetic flux should be less than the inverse single-qubit Josephson energy (less than a nanosecond), which is a challenge for present experiments. The third proposal requires additional subcircuits, e.g., in Refs. [12,13]. These additional elements increase the complexity of the circuits and add additional uncontrollable noise.

To easily switch on and off the coupling among qubits is one of the most important open problems in quantum information hardware. Here, we propose a way to overcome this severe problem plaguing experiments. Specifically, we present a proposal on how to achieve a controllable interaction between flux qubits by virtue of variable frequency or time-dependent magnetic fluxes (TDMFs). Here, we make the same assumption as in the decoupling experiments [5-7], which require the two qubits to be in the large detuning regime. However, here, the two-qubit coupling and decoupling are controlled by the frequency (not the dc component) of the applied TDMF. So we completely avoid having to quickly change the bias magnetic flux - a severe problem faced by many previous proposals for superconducting qubits. Moreover, our proposal does not require additional subcircuits. These merits also make our proposal potentially useful for a variety of other types of qubit experiments, and could solve a central problem in this field.

Controllable Hamiltonian. - Two flux qubits interact with each other through a mutual inductance $M$, as shown in Fig. 1. Each qubit loop contains three junctions, one of them has an area $\alpha$ times smaller than that of the two identical junctions. The larger junction in the $l$ th qubit loop has Josephson energy $E_{\mathrm{J}, l}(l=1,2)$. The gauge-invariant phases (of the two identical junctions and the smaller one) in the $l$ th qubit are $\varphi_{1}^{(l)}, \varphi_{2}^{(l)}$, and $\varphi_{3}^{(l)}$. We assume that a static (dc) magnetic flux $\Phi_{e}^{(l)}$ and a TDMF $\Phi_{e}^{(l)}(t)=$ $A_{l} \cos \left(\omega_{c}^{(l)} t\right)$ are applied through the $l$ th qubit. Using the phase constraint condition through the $l$ th qubit loop $\sum_{i=1}^{3} \varphi_{i}^{(l)}+\left(2 \pi \Phi_{e}^{(l)} / \Phi_{0}\right)+\left[2 \pi \Phi_{e}^{(l)}(t) / \Phi_{0}\right]=0$, the total

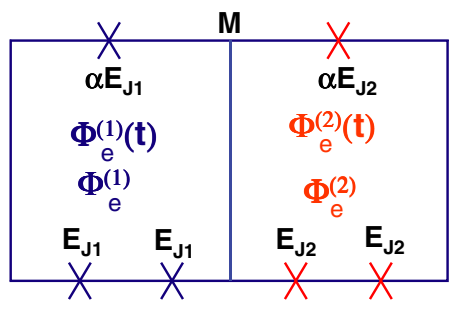

FIG. 1 (color online). Two superconducting flux qubits are coupled through their mutual inductance $M$. Each qubit loop includes three junctions. The tunable interaction between two qubits can be realized by changing the frequency of the external magnetic flux $\Phi_{e}^{(l)}(t)(l=1,2)$ through the $l$ th qubit. 
Hamiltonian of the two qubits can be written as [14]

$$
H=\sum_{l=1}^{2}\left(H_{l}+H_{\mathrm{D}}^{(l)}\right)+\sum_{l \neq m=1}^{2} H_{l m}+H_{C}+H_{A} .
$$

Here, the single-qubit Hamiltonian $H_{l}$ is $H_{l}=\left[P_{P, l}^{2} /\right.$ $\left.\left(2 M_{P, l}\right)\right]+\left[P_{Q, l}^{2} /\left(2 M_{Q, l}\right)\right]+2 E_{\mathrm{J}, l}\left(1-\cos \varphi_{Q}^{(l)} \cos \varphi_{P}^{(l)}\right)+$ $\alpha E_{\mathrm{J}, l}\left[1-\cos \left(2 \varphi_{P}^{(l)}+2 \pi f_{l}\right)\right]$ with the redefined phases $\varphi_{P}^{(l)}=\left(\varphi_{1}^{(l)}+\varphi_{2}^{(l)}\right) / 2, \varphi_{Q}^{(l)}=\left(\varphi_{1}^{(l)}-\varphi_{2}^{(l)}\right) / 2$, and reduced bias magnetic flux $f_{l}=\Phi_{e}^{(l)} / \Phi_{0}$. The effective masses are $M_{Q, l}=2\left(\Phi_{0} / 2 \pi\right)^{2} C_{\mathrm{J}, l}$ and $M_{P, l}=(1+2 \alpha) M_{Q, l}$, which correspond to the effective momenta $P_{Q, l}=$ $-i \hbar\left(\partial / \partial \varphi_{Q}^{(l)}\right)$ and $P_{P, l}=-i \hbar\left(\partial / \partial \varphi_{P}^{(l)}\right)$. The capacitances in the $l$ th qubit loop satisfy the condition $C_{1}^{(l)}=C_{2}^{(l)}=C_{\mathrm{J}, l}$ and $C_{3}^{(l)}=\alpha C_{\mathrm{J}, l}$. The Hamiltonian $H_{\mathrm{D}}^{(l)}=-\left(A_{l} / 2\right)\left(I_{3}^{(l)}+\right.$ $\left.i \beta P_{P, l}\right) e^{-i \omega_{c}^{(l)} t}+$ H.c. represents the interaction between the $l$ th qubit and its TDMF. Here, the parameter $\beta=$ $2 \pi \alpha \omega_{c}^{(l)} /\left[\Phi_{0}(1+2 \alpha)\right], \quad$ and $\quad I_{3}^{(l)}=-\left(2 \pi \alpha E_{\mathrm{J}, l} / \Phi_{0}\right) \times$ $\sin \left(2 \varphi_{P}^{(l)}+2 \pi f_{l}\right)$ is the supercurrent through the smaller junction of the $l$ th qubit without applying the TDMF. So a TDMF-controlled single-qubit rotation can be realized by the Hamiltonian $H_{\mathrm{D}}^{(l)}$. The qubit-qubit interaction $H_{l m}$, controlled by one of the TDMFs $\left[\Phi_{e}^{(1)}(t)\right.$ or $\left.\Phi_{e}^{(2)}(t)\right]$, can be described by $H_{l m}=-\beta_{l} I^{(m)} e^{-i \omega_{c}^{(l)} t} \cos \left(2 \varphi_{P}^{(l)}+\right.$ $\left.2 \pi f_{l}\right)+$ H.c., where $\beta_{l}=M\left(2 \pi / \Phi_{0}\right)^{2}\left(A_{l} C_{l} E_{\mathrm{J}, l} / 2 C_{\mathrm{J}, l}\right)$, and $I^{(m)}=C_{m} \sum_{i}\left(I_{0 i}^{(m)} / C_{i}^{(m)}\right) \sin \varphi_{i}^{(m)}$ is the qubit loop current of the $m$ th qubit without an applied TDMF, and $C_{m}^{-1}=$ $\sum_{i=1}^{3}\left(C_{i}^{(m)}\right)^{-1}$. However, the qubit-qubit interaction $H_{C}$, controlled by simultaneously applying two TDMFs $\left[\Phi_{e}^{(1)}(t)\right.$ and $\left.\Phi_{e}^{(2)}(t)\right]$ through the two qubits, respectively, is $H_{C}=B \prod_{l=1}^{2} \Phi_{e}^{(l)}(t) \cos \left(2 \varphi_{p}^{(l)}+2 \pi f_{l}\right)$, with $B=$ $M\left(2 \pi / \Phi_{0}\right)^{4}\left(C_{1} C_{2} E_{\mathrm{J}, 1} E_{\mathrm{J}, 2} / C_{\mathrm{J}, 1} C_{\mathrm{J}, 2}\right)$. The Hamiltonian $H_{A}=M I^{(1)} I^{(2)}$ denotes an always-on interaction between the two flux qubits, without applying the TDMF.

In the two-qubit basis $\left\{\left|e_{1}\right\rangle,\left|g_{1}\right\rangle\right\} \otimes\left\{\left|e_{2}\right\rangle,\left|g_{2}\right\rangle\right\}$, where $\left|g_{l}\right\rangle$ and $\left|e_{l}\right\rangle$ are the two lowest eigenstates (ground and first excited states) of $H_{l}$, Eq. (1) can become [15]

$$
\begin{aligned}
H= & \sum_{l=1}^{2} \frac{1}{2} \hbar \omega_{l} \sigma_{z}^{(l)}-\sum_{l=1}^{2}\left(\kappa_{l} \sigma_{+}^{(l)} e^{-i \omega_{c}^{(l)} t}+\text { H.c. }\right) \\
& -\sum_{l \neq m=1}^{2}\left(\Omega_{l m}^{(1)} \sigma_{+}^{(l)} \sigma_{-}^{(m)}+\text { H.c. }\right)\left(e^{i \omega_{c}^{(l)} t}+e^{-i \omega_{c}^{(l)} t}\right) \\
& -\sum_{l \neq m=1}^{2}\left(\Omega_{l m}^{(2)} \sigma_{+}^{(l)} \sigma_{+}^{(m)} e^{-i \omega_{c}^{(l)} t}+\text { H.c. }\right) \\
& +\left(\lambda_{1} \sigma_{+}^{(1)} \sigma_{-}^{(2)}+\lambda_{2} \sigma_{+}^{(1)} \sigma_{+}^{(2)}+\text { H.c. }\right) .
\end{aligned}
$$

Here, the terms $\kappa_{l}^{*} \sigma_{+}^{(l)} e^{i \omega_{c}^{(l)} t}$ and $\Omega_{l m}^{(2)} \sigma_{+}^{(l)} \sigma_{+}^{(m)} e^{i \omega_{c}^{(l)} t}$, as well as their complex conjugates, have been neglected by considering energy conservation. The operators of the $l$ th qubit are defined as $\sigma_{z}^{(l)}=\left|e_{l}\right\rangle\left\langle e_{l}|-| g_{l}\right\rangle\left\langle g_{l}\left|, \sigma_{+}^{(l)}=\right| e_{l}\right\rangle \times$ $\left\langle g_{l}\right|$, and $\sigma_{-}^{(l)}=\left|g_{l}\right\rangle\left\langle e_{l}\right|$. The qubit frequency $\omega_{l}$ in Eq. (2) can be expressed as $\omega_{l}=\sqrt{2 I^{(l)}\left(\Phi_{e}^{(l)}-\Phi_{0} / 2\right)^{2}+t_{l}^{2}}$ with the loop current $I^{(l)}$ and the bias flux $\Phi_{e}^{(l)}$. Here, the parameter $t_{l}$ denotes the tunnel coupling between two wells in the $l$ th qubit [16]. The controllable coupling constants are $\kappa_{l}=A_{l}\left\langle e_{l}\left|\left(I_{3}^{(l)}+i \beta P_{P, l}\right)\right| g_{l}\right\rangle / 2, \quad \Omega_{l m}^{(1)}=$ $A_{l} \beta_{l}\left\langle e_{l}, g_{m}\left|I^{(m)} \cos \left(2 \varphi_{P}^{(l)}+2 \pi f_{l}\right)\right| g_{l}, e_{m}\right\rangle / 2$, and $\Omega_{l m}^{(2)}=$ $A_{l} \beta_{l}\left\langle e_{l}, e_{m}\left|I^{(m)} \cos \left(2 \varphi_{P}^{(l)}+2 \pi f_{l}\right)\right| g_{l}, g_{m}\right\rangle / 2$. The phases and amplitudes of $\kappa_{l}, \Omega_{l m}^{(1)}$, and $\Omega_{l m}^{(2)}$ can be controlled by the applied TDMFs. The hard-to-control parameters are $\lambda_{1}=M\left\langle e_{1}, g_{2}\left|I^{(1)} I^{(2)}\right| g_{1}, e_{2}\right\rangle, \quad$ and $\lambda_{2}=$ $M\left\langle e_{1}, e_{2}\left|I^{(1)} I^{(2)}\right| g_{1}, g_{2}\right\rangle$. It is not difficult to derive that $\kappa_{l}=\Omega_{l m}^{(1)}=\Omega_{l m}^{(2)}=0$ for no TDMF. Then, since both bias magnetic fluxes $f_{l}$ are near $f_{l} \approx 1 / 2$ (the optimal point is at $f_{l}=1 / 2$ ), the Hamiltonian (2) can revert to the case in Refs. [3,6], where the Pauli operators are defined by the states of the two potential wells.

Decoupling mechanism and logic gates. -We assume that the two qubits work at the fixed frequencies $\omega_{1}$ and $\omega_{2}$, which mean that their reduced bias magnetic fluxes $f_{i}(i=1,2)$ and the frequency difference $\Delta=\omega_{1}-\omega_{2}$ remain fixed. If the detuning $\Delta$ is initially chosen to be sufficiently large (such that it satisfies the condition: $\left.|\Delta| \gg\left|\lambda_{1}\right| / \hbar=\left|\lambda_{2}\right| / \hbar=|\lambda| / \hbar\right)$, then the two qubits can be approximately treated as two decoupled subsystems [7] when the TDMFs are not applied.

By applying the TDMF with the frequency-matching condition $\omega_{c}^{(l)}=\omega_{l}$, we can easily derive from Eq. (2) that any single-qubit operation of the $l$ th qubit can be performed via the dynamical evolution $U_{c}^{(l)}\left(\theta_{l}, \phi_{l}\right)=$ $\exp \left[i \theta_{l}\left(e^{-i \phi_{l}} \sigma_{-}^{(l)}+\right.\right.$ H.c. $\left.)\right]$. Here, $\theta_{l}=\left|\kappa_{l}\right| \tau / \hbar$ depends on the Rabi frequency $\left|\kappa_{l}\right| / \hbar$ and duration $\tau ; \phi_{l}$ is related to the TDMF phase applied to the $l$ th qubit. For example, $\pi / 2$ rotations of the $l$ th qubit around the $x$ or $y$ axes can be performed by $U_{c}^{(l)}\left(\theta_{l}, \phi_{l}\right)$, with $\tau=\hbar \pi / 4\left|\kappa_{l}\right|$ and $\phi_{l}=\pi$ or $\pi / 2$. Unless specified otherwise, hereafter we work in the interaction picture, and all nonresonant terms have been neglected because their contributions to the transitions between different states are negligibly small [17].

To couple two qubits with the assistance of the TDMF: (i) a TDMF needs to be applied through one of the qubits, and its frequency should be equal to the detuning (or sum) of the two-qubit frequencies; (ii) the reduced bias flux [18] on the qubit, which is addressed by the TDMF, should be near but not equal to $1 / 2$. Without loss of generality, below, the TDMF is assumed to be always applied through the first qubit, so the bias of the first qubit is $f_{1}=1 / 2 \pm \epsilon$, with small $\epsilon$; however, the bias for the second qubit is taken as $f_{2}=1 / 2$.

Considering two new frequency-matching conditions in Eq. (2) produces two different kinds of Hamiltonians for implementing two-qubit operations with the assistance of 
TDMF. One is $H_{1}=\Omega_{12}^{(1)} \sigma_{+}^{(1)} \sigma_{-}^{(2)}+$ H.c., with the condition: $\omega_{1}-\omega_{2} \pm \omega_{c}^{(1)}=0$. Here, the sign is positive (negative) when $\Delta<0 \quad(\Delta>0)$. Another one is $H_{2}=\Omega_{12}^{(2)} \sigma_{+}^{(1)} \sigma_{+}^{(2)}+$ H.c., when the frequencies satisfy the condition: $\omega_{1}+\omega_{2}-\omega_{c}^{(1)}=0$. Using the Hamiltonian $H_{1}$ and $H_{2}$, two-qubit gates can be implemented. For example, a TDMF is applied through the first qubit with its frequency $\omega_{c}^{(1)}$, satisfying the condition $\omega_{1}-$ $\omega_{2}-\omega_{c}^{(1)}=0$ (hereafter we assume $\omega_{1}>\omega_{2}$ without loss of generality). The Hamiltonians $H_{1}$ can be transformed to $H_{1}=H_{X Y}=-\left(\left|\Omega_{12}^{(1)}\right| / 2\right)\left(\sigma_{x}^{(1)} \sigma_{x}^{(2)}+\sigma_{y}^{(1)} \sigma_{y}^{(2)}\right)$, when the phase of $\Omega_{12}^{(1)}$ is set to $\pi$ by an applied TDMF. Then an ISWAP gate [19], denoted by $U_{\mathrm{iS}}$, can be realized by $H_{X Y}$ with an evolution time $t=\pi \hbar /\left(2\left|\Omega_{12}^{(1)}\right|\right)$. The CNOT gate can be constructed by combining the ISWAP gate with a few single-qubit operations.

Experimentally, it is found that the always-on coupling strength $|\lambda| / h$ is about several hundred MHz, e.g., $|\lambda| / h \sim$ $0.4 \mathrm{GHz}$ in Refs. [3,6]; the detuning $\Delta / 2 \pi$ can be $\sim 1$ to $10 \mathrm{GHz}$. This means that the ratio $|\lambda| / \hbar \Delta$ cannot be infinitesimally small, and the always-on interaction needs to be considered when all TDMFs are switched off. Up to the first order in $|\lambda| / \hbar \Delta$, the effect of the always-on interaction, without the TDMF, can be described by an effective Hamiltonian $H_{E}=\left(|\lambda|^{2} / \hbar \Delta\right)\left[\left|e_{1}\right\rangle \times\right.$ $\left.\left\langle e_{1}|\otimes| g_{2}\right\rangle\left\langle g_{2}|-| g_{1}\right\rangle\left\langle g_{1}|\otimes| e_{2}\right\rangle\left\langle e_{2}\right|\right]$. Here, the fast oscillating terms have been neglected.

Entangled states and tomographic measurements. Entangled states can be easily generated in this circuit. For example, if the first and second qubits are initially in the excited state $\left|e_{1}\right\rangle$ and the ground state $\left|g_{2}\right\rangle$, then using the Hamiltonian $H_{1}$, the system evolves to $\left|\Psi_{1}(t)\right\rangle=$ $\cos \left(\left|\Omega_{12}^{(1)}\right| t / \hbar\right)\left|e_{1}, g_{2}\right\rangle-i e^{-i \theta} \sin \left(\left|\Omega_{12}^{(1)}\right| t / \hbar\right)\left|g_{1}, e_{2}\right\rangle \quad$ with the phase $\theta$ of $\Omega_{12}^{(1)}$. It is obvious that the Bell states $\left|\psi_{ \pm}\right\rangle=\left(\left|g_{1}, e_{2}\right\rangle \pm\left|e_{1}, g_{2}\right\rangle\right) / \sqrt{2}$ can be generated with $t_{1}=\hbar \pi /\left(4\left|\Omega_{12}^{(1)}\right|\right)$ by setting the TDMF such that $\theta=$ $\pi / 2$ or $3 \pi / 2$. Similarly, if both qubits are in the ground states $\left|g_{1}\right\rangle$ and $\left|g_{2}\right\rangle$, then another two Bell states $\left|\Psi_{ \pm}\right\rangle=$ $\left(\left|g_{1}, g_{2}\right\rangle \pm\left|e_{1}, e_{2}\right\rangle\right) / \sqrt{2}$ can also be obtained with $t_{2}=$ $\hbar \pi /\left(4\left|\Omega_{12}^{(2)}\right|\right)$ by setting the phase $\theta^{\prime}$ of $\Omega_{12}^{(2)}$ as $\pi / 2$ or $3 \pi / 2$ through the Hamiltonian $\mathrm{H}_{2}$.

State tomography allows us to experimentally determine a quantum state [20]. Qubit state tomography can be implemented by measuring the supercurrent through the qubit loop, which is inductively coupled to, e.g., a dc SQUID magnetometer or high-quality tank circuit [3]. For the $l$ th qubit in the qubit basis $\left\{\left|g_{l}\right\rangle,\left|e_{l}\right\rangle\right\}$, its loopcurrent operator can be written [21] as

$$
\hat{I}^{(l)} \equiv \hat{I}_{x}^{(l)}=a_{l} \sigma_{z}^{(l)}+b_{l}\left|e_{l}\right\rangle\left\langle g_{l}\left|+b_{l}^{*}\right| g_{l}\right\rangle\left\langle e_{l}\right|
$$

with $a_{l}=\left\langle e_{l}\left|I^{(l)}\right| e_{l}\right\rangle$ and $b_{l}=\left\langle e_{l}\left|I^{(l)}\right| g_{l}\right\rangle$, when the bias $f_{l}$ is near (but not equal to) $1 / 2$. However, at the optimal point $f_{l}=1 / 2$, the supercurrent operator in Eq. (3) can be reduced [21] to $\hat{I}^{(l)} \equiv \hat{I}_{x}^{(l)}=b_{l} \sigma_{x}^{(l)}$, with a real number $b_{l}$ and the Pauli operator $\sigma_{x}^{(l)}=\left|e_{l}\right\rangle\left\langle g_{l}|+| g_{l}\right\rangle\left\langle e_{l}\right|$.

If the simultaneous joint measurement of two qubits can be performed in flux qubit circuits as in phase circuits [7], then single-qubit operations are enough to realize the 15 different measurements [20] on the two-qubit states $\rho=$ $(1 / 4) \sum_{i, j} r_{i, j} \sigma_{i}^{(1)} \otimes \sigma_{j}^{(2)}$, with the Pauli operators $\sigma_{i}^{(l)}$ $(i, j=x, y, z$ and $l=1,2)$ and the identity operator $\sigma_{0}^{(l)}$, where $r_{00}=1$ by normalization. The loop-current operator for the first qubit is given in Eq. (3) by setting $l=1$ due to the assumption $f_{1} \neq 1 / 2$, but it is reduced to $I_{x}^{(2)}=b_{2} \sigma_{x}^{(2)}$ for the second qubit with $f_{2}=1 / 2$. So the 15 measurements on state $\rho$ are given as $I_{i}^{(1)}$ and $b_{2} \sigma_{j}^{(2)}$ (denoted as single-qubit measurements), as well as $b_{2} I_{i}^{(1)} \otimes \sigma_{j}^{(2)}$ (called two-qubit or joint measurements), with $i, j=x, y, z, I_{y}^{(1)}=$ $Y_{1}^{\dagger} I_{x}^{(1)} Y_{1}$, and $I_{z}^{(1)}=Z_{1}^{\dagger} I_{x}^{(1)} Z_{1}$. It is clear that three measurements $\left(I_{x}^{(1)}, b_{2} \sigma_{x}^{(2)}\right.$, and $\left.b_{2} I_{x}^{(1)} \otimes \sigma_{x}^{(2)}\right)$ on the input twoqubit state $\rho$ can be directly performed. Other 12 measurements can be equivalently obtained by measuring $\left(I_{x}^{(1)}\right.$, $b_{2} \sigma_{x}^{(2)}$, or both of them at the same time) on the rotated state $\rho$. For example, $\pi / 2$ single-qubit rotations $Y_{1}$ around the $y$ axis for the first qubit and $Z_{2}$ around the $z$ axis for the second qubit are simultaneously performed on the state $\rho$, then the measurement $b_{2} I_{x}^{(1)} \otimes \sigma_{x}^{(2)}$ on the rotated state $Y_{1} Z_{2} \rho Z_{2}^{\dagger} Y_{1}^{\dagger}$ is equivalent to the measurement $b_{2} I_{y}^{(1)} \otimes$ $\sigma_{y}^{(2)}$ on the original state $\rho$. Similarly, other joint measurements can also be obtained. Finally, for the 15 measured results, we solve a set of equations for the parameters $r_{i j}$, and a two-qubit state is reconstructed.

If only a single-qubit measurement can be made at a time (besides the six single-qubit measurements mentioned above), a suitable nonlocal two-qubit operation [20] is required to obtain the coefficients (e.g., $r_{y, z}$ ) of the nine joint measurements on the state $\rho$. Here, this is an ISWAP gate $U_{\mathrm{iS}}$, which can be implemented as described above. For example, if an operation $U_{\text {is }}$ is made on the input state $\rho$, then the loop current of the second qubit should be $\left\langle I_{x}^{(2)}\right\rangle=\operatorname{Tr}\left(U_{\mathrm{iS}} \rho U_{\mathrm{iS}}^{\dagger} I_{x}^{(2)}\right)=-b_{2} \operatorname{Tr}\left(\rho \sigma_{y}^{(1)} \otimes \sigma_{z}^{(2)}\right)=-b_{2} r_{y, z}$, and the coefficient $r_{y, z}$ is determined. Combining the ISWAP gate and single-qubit operations for two qubits, the coefficients of the other eight joint measurements can be determined by only measuring the loop current $I_{x}^{(2)}$ of the second qubit. Then, the tomographic measurement on a two-qubit state is completed by single-qubit measurements.

Tomographically measured states are different for completely decoupled (CD) and large detuning (LD) two-qubit systems after two-qubit states are created, if we consider a duration $t$ before measuring the generated two-qubit states. As an example, a schematic representation of a Bell state 

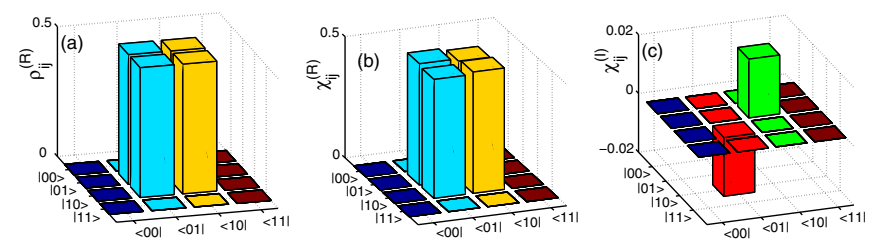

FIG. 2 (color online). A schematic representation of tomographically measured state $\rho$ [with only real part in (a)] for a completely decoupled system, as well as a state $\chi$ [with real part in (b) and imaginary part in (c)] for a two-qubit system with large detuning — after considering a duration $t$ before measuring the created state $\left|\psi_{+}\right\rangle$.

$\left|\psi_{+}\right\rangle$is given in Fig. 2 for the above two cases. There is only the real part for the reconstructed state $\rho=\left|\psi_{+}\right\rangle\left\langle\psi_{+}\right|$ in the CD system, shown in Fig. 2(a). However, due to the effect of $H_{E}$ on the LD system, the reconstructed state $\chi=$ $e^{-i H_{E} t / \hbar}\left|\psi_{+}\right\rangle\left\langle\psi_{+}\right| e^{i H_{E} t / \hbar}$ includes both real and imaginary parts, shown in Figs. 2(b) and 2(c), respectively. In Fig. 2, we consider a longer duration $t \sim 10^{-9} \mathrm{~s}$; the detuning and the coupling constant are, e.g., $\Delta \sim 5 \mathrm{GHz}$, and $|\lambda| / h \sim$ $0.4 \mathrm{GHz}$. So if we consider the always-on interaction effect, the relative error with these parameters is $\sim 0.08$ for the nondiagonal parts of the reconstructed $\mathrm{CD}$ state $\left|\psi_{+}\right\rangle$. Here, the qubit free evolution is neglected. In practice, considering unavoidable environmental effects and statistical errors, the experimentally measured data should be further optimized by other methods [22].

Conclusions. - The controllable coupling of two inductively coupled flux qubits can be realized, when the large detuning condition is satisfied, by the frequency of the TDMF matching or mismatching to the detuning (or sum) of the two-qubit frequencies; not by changing qubit biases (e.g., as in Ref. [7]). Our proposal is also different from the coupling/decoupling method by using dressed states [23]. We emphasize that the deviation $\epsilon$ from the optimal point $1 / 2$ for the reduced bias $f_{1}$ through the first qubit will make the decoherence time $T_{2}$ short. However, our proposal can work for a small deviation, e.g., $\epsilon \sim$ $10^{-4}$, in which $T_{2} \gtrsim 20 \mathrm{~ns}$ in Ref. [24] or $T_{2} \gtrsim 100 \mathrm{~ns}$ with spin-echo signals [24]. At this point, the qubit coupling constant $\Omega_{12}^{(i)}(i=1,2)$ can reach [18] about several hundred MHz. Based on numerical estimates [21], the longest time for the single-qubit operation $Z_{l}$ is less than $5 \mathrm{~ns}$, so the tomographic measurements can be performed within $T_{2}$. Our proposal can be scalable to a chain of many inductively coupled flux qubits, if all of qubits satisfy the large detuning condition. We need to note: (i) we can use one LC circuit as a common information bus to couple many qubits, with the qubit-bus coupling controlled by externally variable frequencies [25]; (ii) this circuit can be modified to work at the optimal point; (iii) this method using frequency-controlled couplings can be applied to control one-junction flux qubits. It can also be modified to control phase, charge-flux, and charge qubits.

We thank J. Q. You, Y. Nakamura, Y.A. Pashkin, O. Astafiev, and K. Harrabi for helpful discussions. This work was supported in part by the NSA and ARDA under AFOSR Contract No. F49620-02-1-0334, and by the NSF Grant No. EIA-0130383.

[1] J. Q. You and F. Nori, Phys. Today 58 No. 11, 42 (2005).

[2] Y.A. Pashkin et al., Nature (London) 421, 823 (2003); T. Yamamoto et al., ibid. 425, 941 (2003).

[3] A. Izmalkov et al., Phys. Rev. Lett. 93, 037003 (2004); M. Grajcar et al., Phys. Rev. B 72, 020503(R) (2005).

[4] H. Xu et al., Phys. Rev. Lett. 94, 027003 (2005).

[5] A. J. Berkley et al., Science 300, 1548 (2003).

[6] J. B. Majer et al., Phys. Rev. Lett. 94, 090501 (2005).

[7] R. McDermott et al., Science 307, 1299 (2005).

[8] A. Blais et al., Phys. Rev. Lett. 90, 127901 (2003); A. Blais et al., Phys. Rev. A 69, 062320 (2004).

[9] A. N. Cleland and M.R. Geller, Phys. Rev. Lett. 93, 070501 (2004).

[10] Y. Makhlin et al., Rev. Mod. Phys. 73, 357 (2001).

[11] J. Q. You et al., Phys. Rev. Lett. 89, 197902 (2002); Y. X. Liu et al., Europhys. Lett. 67, 941 (2004); L. F. Wei et al., ibid. 67, 1004 (2004); Phys. Rev. B 71, 134506 (2005).

[12] D. V. Averin and C. Bruder, Phys. Rev. Lett. 91, 057003 (2003).

[13] B.L. T. Plourde et al., Phys. Rev. B 70, 140501(R) (2004).

[14] Here, we neglect the self-interaction term of the external magnetic fields $H_{\text {self }}=\sum_{l=1}^{2}\left(\alpha C_{J}^{(l)} / 2\right)\left[\partial \Phi_{e}^{(l)}(t) / \partial t\right]^{2}$. The self-inductance energy terms for two qubits are also neglected here; these terms do not affect our predictions.

[15] In the derivation of Eq. (2), we have discarded the two-field-controlled qubit-qubit interaction term $H_{C}=$ $B\left(\chi_{1} \sigma_{+}^{(1)}+\chi_{1}^{*} \sigma_{-1}^{(1)}\right)\left(\chi_{2} \sigma_{+}^{(2)}+\chi_{2}^{*} \sigma_{-}^{(2)}\right) \Phi_{e}^{(1)}(t) \Phi_{e}^{(2)}(t) \quad$ with $\chi_{l}=\left\langle 1\left|\cos \left(2 \varphi_{p}^{(l)}+2 \pi f_{l}\right)\right| 0\right\rangle(l=1,2)$. In principle, the qubit-qubit interaction can also be controlled by two external fields, one for each qubit loop. However, one field is enough to achieve our goal.

[16] J. E. Mooij et al., Science 285, 1036 (1999); T. P. Orlando et al., Phys. Rev. B 60, 15398 (1999).

[17] W.H. Louisell, Quantum Statistical Properties of Radiation (John Wiley \& Sons, New York, 1973).

[18] Y.X. Liu et al., Phys. Rev. Lett. 95, 087001 (2005).

[19] N. Schuch and J. Siewert, Phys. Rev. A 67, 032301 (2003).

[20] Y. X. Liu et al., Europhys. Lett. 67, 874 (2004); Phys. Rev. B 72, 014547 (2005).

[21] J. Q. You et al., Phys. Rev. B 71, 024532 (2005).

[22] M. Paris and J. Rehacek, Quantum State Estimation, Lecture Notes in Physics Vol. 649 (Springer, Berlin, 2004).

[23] C. Rigetti et al., Phys. Rev. Lett. 94, 240502 (2005).

[24] P. Bertet et al., Phys. Rev. Lett. 95, 257002 (2005).

[25] Y.X. Liu et al., cond-mat/0509236. 